\title{
Numerical Simulations of Nonlinear Interaction of Space Charge Waves in Microwave and Millimeter Wave Range in n-InN Films Using Negative Differential Conductivity
}

\author{
Abel Garcia-Barrientos', Volodymyr Grimalsky² \\ ${ }^{1}$ Electronics and Computer Science Department, Research Center on Information Technology and Systems, \\ Autonomous University of Hidalgo State (UAEH), Hidalgo, México \\ ${ }^{2}$ CIICAp, Autonomous University of Morelos State (UAEM), Morelos, México \\ Email: agarciab@ieee.org
}

Received 14 May 2014; revised 13 June 2014; accepted 14 July 2014

Copyright @ 2014 by authors and Scientific Research Publishing Inc.

This work is licensed under the Creative Commons Attribution International License (CC BY).

http://creativecommons.org/licenses/by/4.0/

(c) (i)

\section{Abstract}

Numerical simulations of nonlinear interaction of space charge waves in microwave and millimeter wave range in $\mathrm{n}$-InN films have been carried out. A micro- and millimeter-waves frequency conversion using the negative differential conductivity phenomenon is carried out when the harmonics of the input signal are generated. An increment in the amplification is observed in $\mathbf{n}$-InN films at essentially at high-frequencies $f<450 \mathrm{GHz}$, when compared with $\mathrm{n}$-GaAs films $\mathrm{f}<44 \mathrm{GHz}$. This work provides a way to achieve a frequency conversion and amplification of micro- and millimeter-waves.

\section{Keywords}

Space Charge Waves, InN Film, Negative Differential Conductivity

\section{Introduction}

Semiconducting group-III nitrides have attracted a lot of attention in the recent decade because of, mainly, the large gap $(0.7-6.2 \mathrm{eV})$ that can be covered by the nitrides and their alloys. Their electrical and optical properties are highly suitable for novel high frequency electronics, optoelectronics and photonics applications. Among 
those, in particular, Indium Nitride (InN) has become the focus of intense research after recent reports of an unexpectedly low band gap of $0.7 \mathrm{eV}$ at room temperature [1] [2]. A great deal of experimental and theoretical effort has been made to better understand the nature of InN, to determine the properties of this material, and to grow high-quality epitaxial $\mathrm{InN}$ layers. InN is a promising material, for colorful display, high efficiency solar cells of low cost and high-frequency electronics for its high electron mobility due to its large drift velocity at room temperature. For high-frequency electronics applications, specially, millimeter and sub-millimeter microwave ranges are very important for applications in communications, radar, meteorology and spectroscopy. However, the structure of semiconductor devices (transistors, diodes, etc.), required for such a short wavelength, becomes very complex, which makes its fabrication difficult and expensive with actual technologies. One potential alternative to explore the use of such a part of the electromagnetic spectrum resides in the use of non-linear wave interaction in an active media. For example, the space charge waves in thin semiconductor films, possessing negative differential conductivity, GaAs [3], InP [4], GaN [5], and InN [6] propagate at frequencies that are higher than the frequencies of acoustic and spin waves in solids. Space charge waves have been researched since a long time ago, which can be traced back to the 1950s [7]. The early experimental work on the amplification of space charge waves with a perturbation field started in the 1970s [7]-[9] and continued today. As pointed out in 1970, in the seminal book by Scott [9] on active and nonlinear wave propagation in electronics, there was, by that time, research stretching back to the 1950s on devices such as traveling-wave tubes and backward-wave oscillators. Devices based on space charge waves use an attractive property of III-Nitride semiconductors, the negative differential conductance. An electric field in excess of $50 \mathrm{kV} / \mathrm{cm}$ applied to an n-InN sample causes the differential electron mobility to become negative. To analyze wave phenomena in thin films of two-valley semiconductors [10], a set of equations to describe the charge transport is commonly used. In this theory, with small initial perturbations, continuity, momentum and energy equations, and Poisson's equation are solved numerically. The solutions show that the modulations of electron density travel along the beam in the form of waves called space charge waves. Although, this paper presents the numerical simulations of amplification and propagation of space charge waves in InN films, the experimental verification can be carried out in the same way like in GaAs films, already reported in Ref. [11].

\section{Electron Transport Model}

Nonlinear interaction of space charge waves in microwave and millimeter wave range in n-InN films using the negative differential conductivity will be one of the most relevant topics in microelectronics and communications in the coming years, due to the potential it represents in terms of amplification of micro- and millimeterwaves. Therefore, we present two-dimensional numerical simulations of propagation and amplification of space charge waves in InN films and microwave frequency conversion under negative differential conductivity phenomenon. We use a high quality, single crystal wurtzita InN film placed onto substrate without a acoustic contact. It is assumed that the electron gas is localized in the center of film. The thickness of the n-InN film is $2 \mathrm{~h} \leq$ $1 \mu \mathrm{m}$, see Figure 1.

The coordinate system is chosen as follows: X-axis is directed perpendicularly to the film, the electric field $E_{0}$

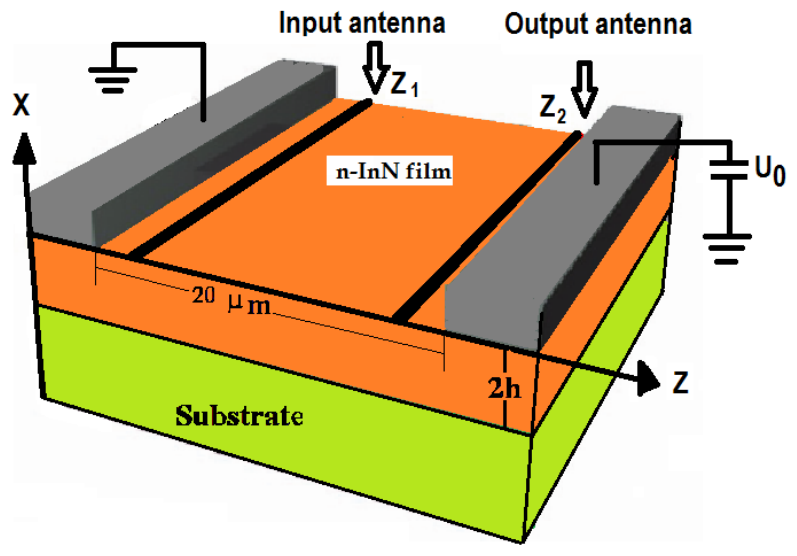

Figure 1. The structure of the n-InN traveling-wave amplifier with an epitaxial layer. 
is applied along Z-axis, exciting and receiving antennas are parallel to $\mathrm{Y}$-axis. 2D model of electron gas in the n-InN film is used. Taking a constant surface sheet density of $2 \mathrm{D} n_{0}=2.5 \times 10^{13} \mathrm{~cm}^{-2}$ for a $1 \mu \mathrm{m}$ thick film. There has been some evidence to suggest the presence of electron accumulation at the surface of InN, including measurements of the sheet carrier density as a function of $\operatorname{InN}$ film thickness and capacitance-voltage profiling [12]. The space charge waves possessing phase velocity equal to drift velocity of the electrons $v_{0}=v\left(E_{0}\right), E_{0}=$ $U_{0} / L_{z}$, are considered, where $U_{0}$ is bias voltage, $L_{\mathrm{z}}$ is the length of the film. Generally, a non-local dependence of drift velocity $v_{d}$ of electrons on the electric field takes place. In simulations, an approximation of two-dimensional electron gas is used. The set of balance equations for concentration, drift velocity, and the averaged energy to describe the dynamics of space charge waves within the n-InN film takes a form, like in GaAs film [10]:

$$
\begin{aligned}
& \frac{\mathrm{d}\left(m(w) \boldsymbol{v}_{d}\right)}{\mathrm{d} t}=-q\left(\boldsymbol{E}-\frac{\boldsymbol{v}_{d} E_{s}}{v_{s}}\right) ; \quad \frac{\mathrm{d} w}{\mathrm{~d} t}=-q\left(\boldsymbol{E} \boldsymbol{v}_{d}-E_{s} v_{s}\right) ; \\
& \frac{\partial n}{\partial t}+\operatorname{div}\left(n \boldsymbol{v}_{d}-D \nabla n\right)=0 ; \quad D(w)=\frac{2}{3} \frac{\tau_{p}(w)}{m(w)}\left(w-\frac{1}{2} m v^{2}\right) ; \\
& \boldsymbol{E}=\boldsymbol{e}_{z} E_{0}-\nabla \varphi+\boldsymbol{e}_{z} \tilde{E}_{e x t} ; \quad \Delta \varphi=\frac{q}{\varepsilon_{0} \varepsilon}\left(n-n_{0}\right) \delta(x) ; \\
& \tilde{E}_{e x t}=\sum_{j=1}^{2} E_{o j} \sin \left(\omega_{j} t\right) \exp \left(-\left(\frac{z-z_{1}}{z_{0}}\right)^{2}-\left(\frac{y-y_{1}}{y_{0}}\right)^{2}\right)
\end{aligned}
$$

where $v_{d}$ is drift velocity, $\varphi$ is the of potential, $n=n_{0}+\tilde{n}$ where $n_{0}$ is constant electron concentration, $\tilde{n}$ is the varying part, $w$ is the electron energy, $D$ is the diffusion coefficient, and $\varepsilon_{0}$ is the lattice dielectric permittivity of $\mathrm{n}$-InN, $m(w)$ is averaged effective mass, $q$ is the electron charge, $\tau_{p, w}(w)$ are relaxation times, $E_{\text {ext }}$ is a small microwave electric signal applied to the input antenna and $E_{0}$ is the bias electric field. It is assumed that a condition of occurring negative differential conductivity is realized. Because the signal frequencies are in microwave or millimeter wave range, it is possible to separate diffusion and drift motions. For the sake of simplicity, instead of relaxation times, the parameter $E_{s}$ is introduced [10]:

$$
\frac{m(w)}{\tau_{p}(w)}=\frac{E_{s}}{v_{s}\left(E_{s}\right)}, \quad \frac{w-w_{0}}{\tau_{w}(w)}=q E_{s} v_{s}\left(E_{s}\right)
$$

In such a representation, the mean energy and mean effective mass of electron are denoted by $w$ and $m(w)$, the equilibrium value of $w$ is $w_{0}$; A direct correspondence between local field dependence and non-local effects is well seen. Because a dependence $E_{s}=E_{s}(w)$ is unique, it is possible to express the parameters $w$ and $v_{s}$ through the value of $E_{s}$. The dependencies of drift velocity, averaged electron energy, and effective mass versus electric field in InN films were calculated by our Monte Carlo procedure, which are pretty similar as experimental results [13]-[15]. Several stochastic mechanisms such as acoustic phonon, polar optical phonon, inter-valley phonon, Coulomb, and piezoelectric scattering are considered and their impact is assessed. The parameter values for the acoustic deformation potential (ADP $\Xi=7.1 \mathrm{eV}$ ), polar-optical phonon scattering ( $\hbar \omega=73 \mathrm{meV}$ or $89 \mathrm{meV}$ ), inter-valley scattering $\left(\hbar \omega_{\mathrm{iv}}=\hbar \omega_{\mathrm{LO}}\right)$, mass density $\left(\rho=6.81 \mathrm{~g} / \mathrm{cm}^{3}\right)$, and static and high frequency dielectric constants $\left(\varepsilon_{s}=15.3\right.$ and $\left.\varepsilon_{\infty}=8.4\right)$ are adopted from [16]. In addition, we study the influence of another set of dielectric constants $\left(\varepsilon_{s}=11.0\right.$ and $\left.\varepsilon_{\infty}=6.7\right)$ recently proposed in [16] in conjunction with the narrow bandgap and lower effective mass. The dependencies of drift velocity, averaged electron energy and averaged electron mass on electric field in InN films are taken from our Monte Carlo simulation results (see Figure 2), which are in good agreement with measured data and the set of parameters used in the simulations are very similar from literature [13]-[16]. The maximum value of drift velocity in $\mathrm{InN}$ is when the electric field is $\sim 40 \mathrm{kV} / \mathrm{cm}$, under these high-field conditions, some of the electrons can acquire enough energy from the electric field to transfer via inter-valley scattering to the upper valley. Since the mobility of electrons in the upper valley is smaller than in lower valley, the conductivity decreases with increasing field, leading to a negative differential resistance (see Figure 2(a)). A comparison of the results for GaAs and $\mathrm{InN}$ films reveals that for the field strength considered most of the electrons in GaAs are in higher valley while in InN less than are transferred. This is due to the difference in the separation of the valleys (0.3 eV in GaAs and 2.9 - $3.9 \mathrm{eV}$ in $\mathrm{InN}$ [17]) in spite of the higher 


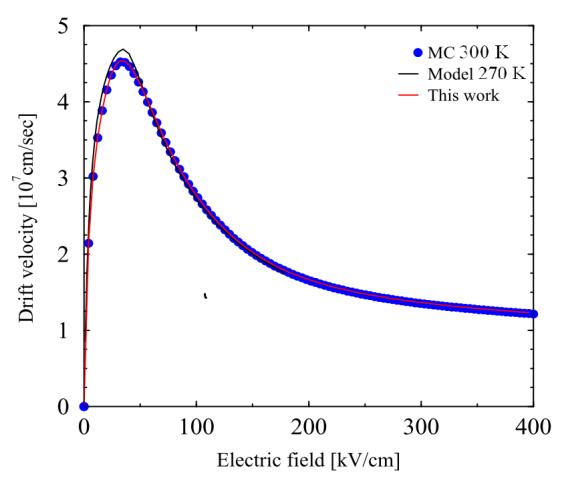

(a)

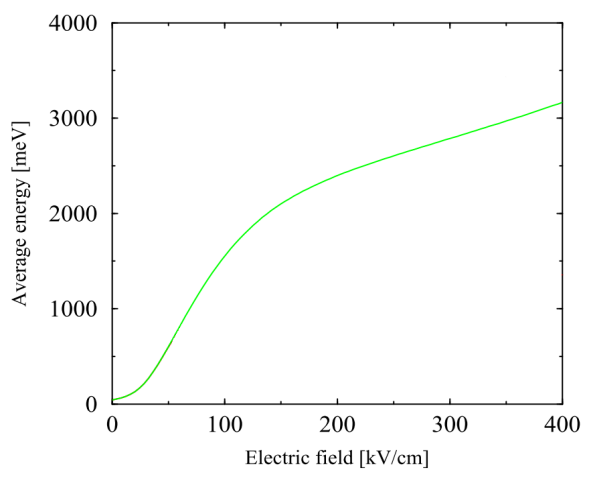

(b)

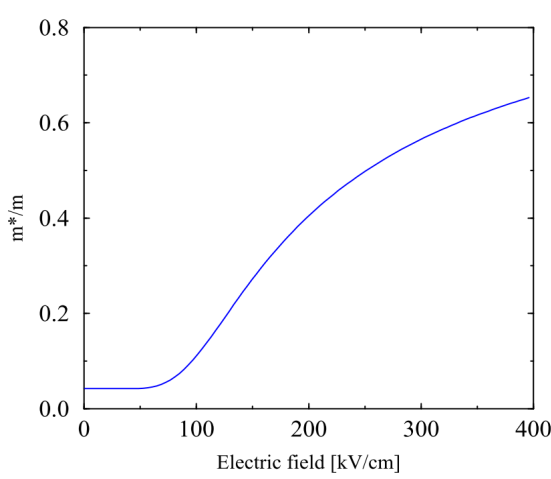

(c)

Figure 2. Electron drift velocity (a), average electron energy (b), averaged mass (c) versus electric field used in simulations.

field applied in InN. A large negative differential conductance appears when increasing electric field more than the threshold fielded; the electrons have not enough energy to make the inter-valley scattering. Beyond this value, the optical scattering mechanisms play a drastic role rather than acoustic and ionized impurity scattering.

Since this process is inelastic, the electron energy curves have sensitive variation in its slope; in fact it does not increase as fast as increasing in initial fields (see Figure 2(b)).

\section{Propagation and Amplification of Space Charge Waves in n-InN Films}

The spatial increment of space charge waves is investigated by the dispersion equation, $\mathrm{D}(\omega, k)=0$, the relation between angular frequency, $\omega=2 \pi f$, and wave vectors, consider these like complex, $k=k^{\prime}+\mathrm{i} k^{\prime \prime}$, where $k^{\prime}>0$. The dispersion relation is obtained using balance equations model, set Equations (1), with some transformation we can obtain Equation (3),

$$
\frac{\partial \tilde{n}}{\partial t}+n_{0} \frac{\partial \tilde{v}}{\partial z}+v_{0} \frac{\partial \tilde{n}}{\partial z}-D \frac{\partial^{2} \tilde{n}}{\partial z^{2}}=0
$$

but if we assume that $\tilde{n}$ obeys the law $\sim \exp (\mathrm{i} \omega t-\mathrm{i} k z)$, Equation (3) gives the dispersion relation:

$$
\left[\mathrm{i}\left(\omega-k v_{0}\right)+D k^{2}\right] \tilde{n}-\mathrm{i} k n_{0} \tilde{v}=0
$$

In general, we consider the cases where $\omega=2 \pi f$ is real and $k=k^{\prime}+\mathrm{i} k^{\prime \prime}$ has real and imaginary part. The case $k^{\prime \prime}>0$ corresponds to spatial increment (amplification), whereas the case $k^{\prime \prime}<0$ corresponds to the decre- ment (damping). In Figure 3(a), the spatial increment of space charge waves in an n-InN film is shown in the curve 3, where the electron concentration is $n_{0}=2.5 \times 10^{13} \mathrm{~cm}^{-2}$, the bias electric field is $E_{0}=50 \mathrm{kV} / \mathrm{cm}$. In curve 2, the electron concentration is $n_{0}=2 \times 10^{13} \mathrm{~cm}^{-2}$ with the same bias electric field, $E_{0}=50 \mathrm{kV} / \mathrm{cm}$. Curve 1 is the result for $\mathrm{n}$-InN films where the electron concentration is $n_{0}=2.5 \times 10^{13} \mathrm{~cm}^{-2}$ and the bias electric field is $E_{0}=45 \mathrm{kV} / \mathrm{cm}$. The stationary values of $E_{0}$ have been chosen in the regime of negative differential conductivity $(\mathrm{d} v / \mathrm{d} E<0)$ for all cases. One can see that an amplification of space charge waves in InN films occurs in a 


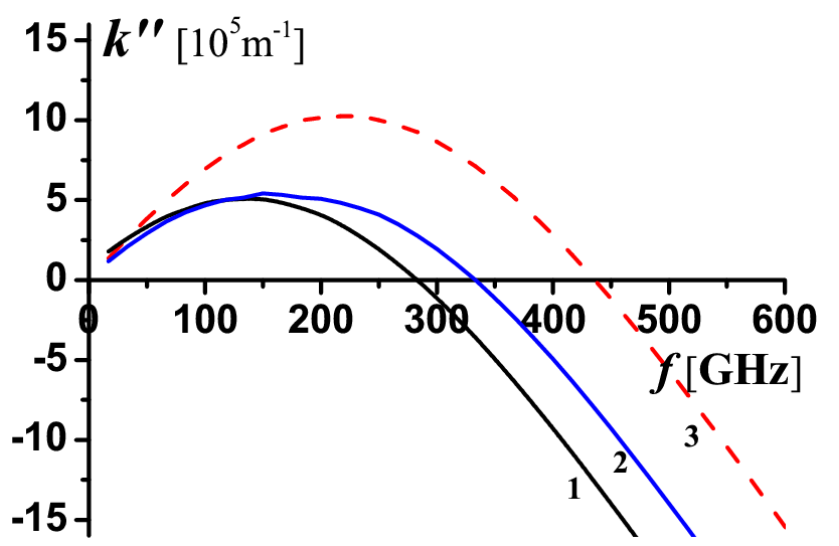

(a)

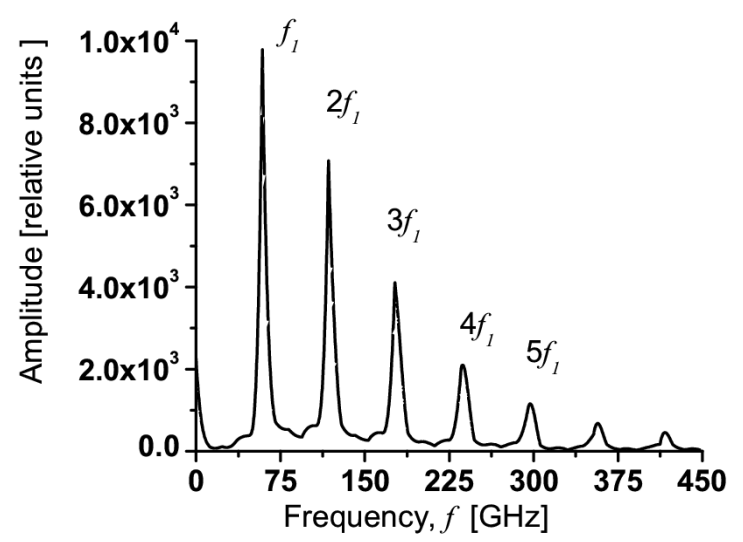

(b)

Figure 3. (a) The spatial increment of space charge waves in an n-InN film is shown in the curve 3, where the electron concentration is $n_{0}=2.5 \times 10^{13} \mathrm{~cm}^{-2}$, the bias electric field is $E_{0}=50 \mathrm{kV} / \mathrm{cm}$. In curve 2, the electron concentration is $n_{0}=2 \times$ $10^{13} \mathrm{~cm}^{-2}$ with the same bias electric field, $E_{0}=50 \mathrm{kV} / \mathrm{cm}$. Curve 1 is the result for $\mathrm{n}$-InN films where the electron concentration is $n_{0}=2.5 \times 10^{13} \mathrm{~cm}^{-2}$ and the bias electric field is $E_{0}=45 \mathrm{kV} / \mathrm{cm}$; (b) Spectral components of the electric field of space charge waves. The effective excitation of harmonics is presented. The input carrier frequency is $f=60 \mathrm{GHz}$.

wide frequency range, and the maximal spatial increment is $k^{\prime \prime}=12 \times 10^{5} \mathrm{~m}^{-1}$ at the frequency $f=225 \mathrm{GHz}$. When compared with a case of the GaAs film [3], it is possible to observe an amplification of space charge waves in InN films at essentially higher frequencies $f>44 \mathrm{GHz}$. To obtain an amplification of $25 \mathrm{~dB}$, it is necessary to use a distance between the input and output antennas of about $19 \mu \mathrm{m}$.

When a small microwave electric signal $E_{e x t}=E_{m} \cdot \sin (\omega t) \cdot \exp \left(-\left(\left(z-z_{1}\right) / z_{0}\right)^{2}-\left(\left(y-y_{1}\right) / y_{0}\right)^{2}\right)$ is ap-

plied to the input antenna. Here $z_{1}$ and $y_{1}$ are the position of the input antenna; $z_{0}$ and $y_{0}$ are its half-width. When this signal is applied, the excitation of space charge waves in 2D electron gas takes place. These waves are subject to amplification, due to negative differential conductivity.

The set of Equations (1) form a set of non-linear coupled time dependent partial differential equations. These differential equations are discretized using a finite-difference scheme and are solved numerically. A transverse inhomogeneity of the structure in the plane of the film along $\mathrm{Y}$ axis is taken into account. The following parameters have been chosen: 2D electron concentration in the film is $n_{0}=2.5 \times 10^{13} \mathrm{~cm}^{-2}$, the initial uniform drift velocity of electrons is $v_{0} \approx 4 \times 10^{7} \mathrm{~cm} / \mathrm{s}\left(E_{0}=50-100 \mathrm{kV} / \mathrm{cm}\right)$, the length of the film is $L_{\mathrm{z}}=5-20 \mu \mathrm{m}$, the thickness of the film is $2 \mathrm{~h}=0.1-1 \mu \mathrm{m}$. The typical output spectrum of the electromagnetic signal is given in Figure 3(b). The input carrier frequency is $f=60 \mathrm{GHz}$. The amplitude of the input electric microwave signal is $E_{m}=25 \mathrm{~V} / \mathrm{cm}$. Although the growth rate decreases as the $r f$ frequency increases, for our case an amplification of $25 \mathrm{~dB}$ is obtained. One can see both the amplified signal at the first harmonic of the input signal and the harmonic generations of the input signal, which is generated due to the non-linearity of space charge waves.

The propagation and amplification of space charge waves in n-GaAs thin films with negative difference conductance have been studied in the last decade [12], however n-InN films have not been addressed yet, and are subject of this work. We address the device presented in Figure 1 by means of numerical simulations. An n-InN epitaxial film of thickness $0.1-1 \mu \mathrm{m}$ is put on an InN semi-insulating substrate. The two-dimensional electron density in the film is chosen to be $n_{0}=2.5 \times 10^{13} \mathrm{~cm}^{-2}$. On the film surface are the cathode and anode ohmiccontacts (OCs), together with the input and output coupling elements (CEs). The CEs connect the sample structure to microwave sources. A $d c$ bias voltage (above the Gunn threshold, $50 \mathrm{kV} / \mathrm{cm}$ ) was applied between the cathode and anode OCs, causing negative differential conductivity in the film. The CEs perform the conversion between electromagnetic waves and space charge waves, where the excitation of space charge waves in the 2D electron gas takes place.

The spatial distributions of the alternate component of the electric field $E_{\mathrm{z}}^{\sim}$ and $E_{\mathrm{y}}^{\sim}$ are shown in Figure 4. One can see the maximum variations are in the output antenna. The length of the film is $20 \mu \mathrm{m}$. The transverse width of the film along Y axis is $40 \mu \mathrm{m}$. The duration of the input electric pulse is $2.5 \mathrm{~ns}$. The spatial distributions are presented for the time moment $1.5 \mathrm{~ns}$ after the maximal value of the input signal. Direct numerical si- 


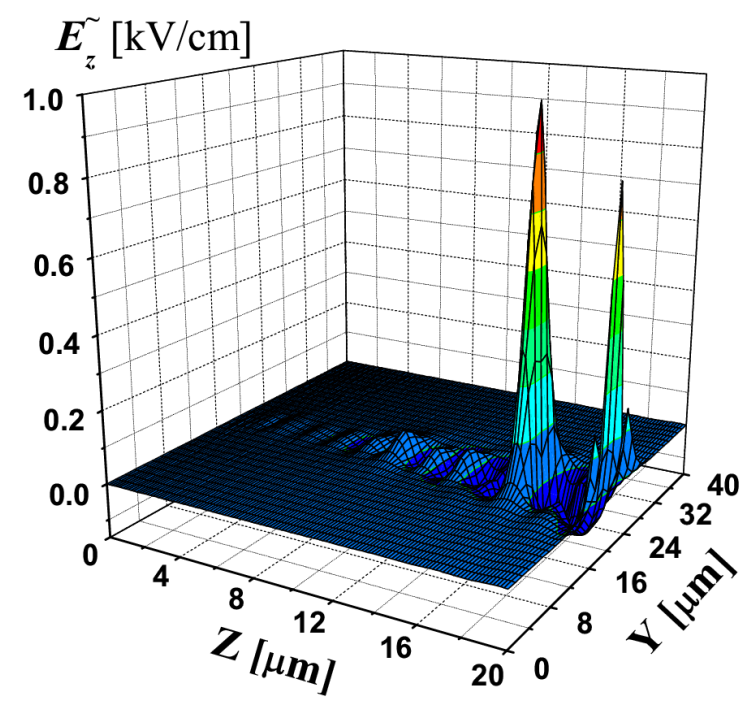

(a)

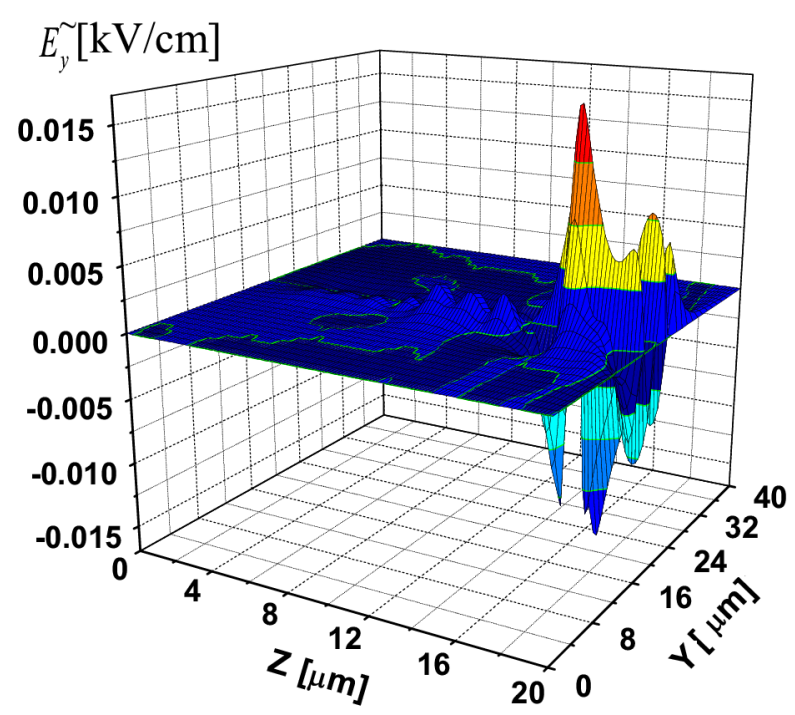

(b)

Figure 4. The spatial distributions of the alternative part of the electric field component ${E^{\sim}}_{\mathrm{z}}$ (a) and ${E_{\mathrm{V}}^{\sim}}_{\mathrm{v}}$ (b) of space charge wave; The length of the film is $20 \mu \mathrm{m}$. The transverse width of the film along Y axis is $40 \mu \mathrm{m}$.

mulations have confirmed pointed below results on linear increments of space charge waves amplification. Also a possibility of non-linear frequency doubling and mixing is demonstrated. To get the effective frequency doubling in the millimeter wave range, it is better to use the films with uniform doping.

\section{Conclusion}

A numerical simulation of nonlinear interaction, two-dimensional propagation and amplification of space charge waves in microwave and millimeter wave range in $\mathrm{n}$-InN films using negative differential conductivity are presented. A microwave frequency conversion using the negative differential conductivity phenomenon is carried out when the harmonics of the input signal are generated. A comparison of the calculated spatial increment of instability of space charge waves in $\mathrm{n}-\mathrm{GaAs}$ and $\mathrm{n}-\mathrm{InN}$ films is performed. An increment in the amplification is observed in n-InN films at essentially higher frequencies $f>44 \mathrm{GHz}$ than in GaAs films [3]. The maximum amplification (gain of $25 \mathrm{~dB}$ ) is obtained at $f=225 \mathrm{GHz}$, using a distance between the input and output antennas of about $19 \mu \mathrm{m}$.

\section{Acknowledgements}

This project has been funded by the CONACyT-Mexico grant CB-169062 and also it has been partially funded by PROMEP: Redes Temáticas de Colaboración under the project titled: Fuentes de Energías Alternas.

\section{References}

[1] Wu, J., Walukiewicz, W., Shan, W., Yu, K.M., Ager III, J.W., Haller, E.E., Lu, H. and Schaff, W.J. (2002) Effects of the Narrow Band Gap on the Properties of InN. Physical Review B, 66, Article ID: 201403. http://dx.doi.org/10.1103/PhysRevB.66.201403

[2] Wu, J., Walukiewicz, W., Yu, K.M., Ager III, J.W., Haller, E.E., Lu, H., Schaff, W.J., Saito, Y. and Nanishi, Y. (2002) Unusual Properties of the Fundamental Band Gap of InN. Applied Physics Letters, 80, 3967-3969.

[3] Garcia, A., Grimalsky, V., Gutierrez, E. and Palankovski, V. (2009) Nonstationary Effects of the Space Charge in Semiconductor Structures. Journal of Applied Physics, 105, Article ID: 074501. http://dx.doi.org/10.1063/1.3093689

[4] Garcia-Barrientos, A. and Palankovski, V. (2011) Numerical Simulations of Space Charge Waves in InP Films and Microwave Frequency Conversion under Negative Differential Conductivity. Applied Physics Letters, 98, 072110-1072110-3.

[5] Koshevaya, S.V., Grimalsky, V.V., Garcia-B, A. and Diaz-A, F. (2012) Amplification and Nonlinear Interaction of Space Charge Waves of Microwave Band in Heterogeneous Gallium Nitride Films. Radioelectronics and Communica- 
tions Systems, 55, 289-298. http://dx.doi.org/10.3103/S0735272712070011

[6] Schley, P., Goldhahn, R., Gobsch, G., Feneberg, M., Thonke, K., Wang, X. and Yoshikawa, A. (2009) Influence of Strain on the Band Gap Energy of Wurtzite InN. Physica Status Solidi (b), 246, 1177-1180. http://dx.doi.org/10.1002/pssb.200880924

[7] Beck, A.H.W. (1958) Space-Charge Waves and Slow Electromagnetic Waves. Pergamon, New York.

[8] Dean, R.H., Dreeben, A.B., Kaminski, J.F. and Triano, A. (1970) Travelling-Wave Amplifier Using Thin Epitaxial GaAs Layer. Electronics Letters, 6, 775-776.

[9] Scott, A. (1970) Active and Nonlinear Wave Propagation in Electronics. John Wiley \& Sons, New York.

[10] Carnez, B., Cappy, A., Kaszynskii, A., Constant, E. and Salmer, G. (1980) Modeling of a Submicrometer Gate FieldEffect Transistor Including Effects of Nonstationary Electron Dynamics. Journal of Applied Physics, 51, 784-790. http://dx.doi.org/10.1063/1.327292

[11] Mikhailov, A.I. (2000) Experimental Study of the Parametric Interaction between Space-Charge Waves in Thin-Film GaAs-Based Semiconductor Structures. Technical Physics Letters, 26, 217-219. http://dx.doi.org/10.1134/1.1262796

[12] Lu, H., Schaff, W.J., Eastman, L.F. and Stutz, C.E. (2003) Surface Charge Accumulation of InN Films Grown by Molecular-Beam Epitaxy. Applied Physics Letters, 82, 1736. http://dx.doi.org/10.1063/1.1562340

[13] Tansley, T. and Foley, C. (1984) Electron Mobility in Indium Nitride. Electronics Letters, 20, 1066-1068. http://dx.doi.org/10.1049/el:19840729

[14] Yamamoto, A., Shin-ya, T., Sugiura, T. and Hashimoto, A. (1998) Electron Mobility in Indium Nitride. Journal of Crystal Growth, 189/190, 461-465. http://dx.doi.org/10.1016/S0022-0248(98)00331-5

[15] Franssen, G., Suski, T., Perlin, P., Teisseyre, H., Khachapuridze, A., Dmowski, L.H., Plesiewicz, J.A., Kaminska, A., Kurouchi, M., Nanishi, Y., Lu, H. and Schaff, W. (2006) Band-to-Band Character of Photoluminescence from InN and In-Rich InGaN Revealed by Hydrostatic Pressure Studies. Applied Physics Letters, 89, Article ID: 121915. http://dx.doi.org/10.1063/1.2356994

[16] Polyakov, V. and Schwierz, F. (2006) Low-Field Electron Mobility in Wurtzite InN. Applied Physics Letters, 88, 032101-1-032101-3. http://dx.doi.org/10.1063/1.2166195

[17] Bougrov, V., Levinshtein, M.E., Rumyantsev, S.L. and Zubrilov, A. (2001) Properties of Advanced Semiconductor Materials GaN, AlN, InN, BN, SiC, SiGe. In: Levinshtein, M.E., Rumyantsev, S.L. and Shur M.S., Eds., John Wiley \& Sons, Inc., New York, 1-30. 
Scientific Research Publishing (SCIRP) is one of the largest Open Access journal publishers. It is currently publishing more than 200 open access, online, peer-reviewed journals covering a wide range of academic disciplines. SCIRP serves the worldwide academic communities and contributes to the progress and application of science with its publication.

Other selected journals from SCIRP are listed as below. Submit your manuscript to us via either submit@scirp.org or Online Submission Portal.
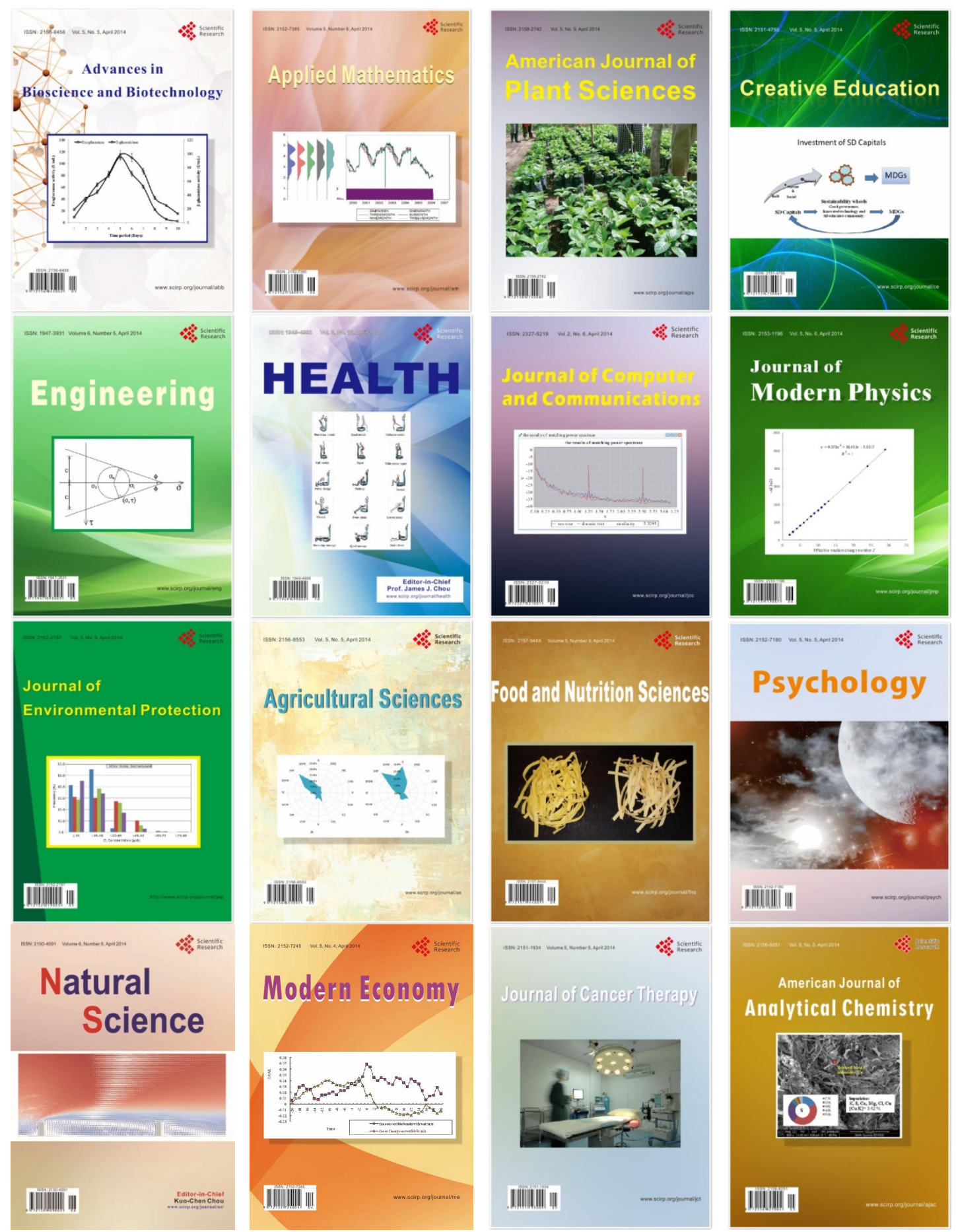